\title{
Two-dimensional numerical tests, of drag compensation device for on-road freight vehicles, based on CFD methods
}

\author{
Martin Ivanov ${ }^{1, *}$ \\ ${ }^{1}$ Technical University - Sofia, FPEPM, Department:" Hydroaerodynamics and Hydraulic Machines", \\ Sofia 1000, Bulgaria
}

\begin{abstract}
In today's demanding reality, making the on-road freight vehicles more fuel-efficient and lower emissive, is becoming more and more important design issue. During the past years the light car industry has managed to reduce significantly the aerodynamic losses, whereas the freight transport did not have much development in this aspect. For heavy vehicles such as tractor-trailers, drag is one of the dominant reasons for energy loss due to poor aerodynamic shape and large surfaces facing the flow direction. That is why, the presented study focuses on the development of technological solution for aerodynamic loss compensation, devoted to the freight transport vehicles. An innovative approach for drag compensation, based on airfoil device located in the tractor-trailer gap, is tested by the methods of the Computational fluid dynamics (CFD), in 2D simulation study. The lift and drag force distribution over the airfoil are analyzed in the restricted area, in relation to the airfoil size. The results show that, the values increase with the increase of the size of the profile. However, important model limitations are described as well, which will assist the further development of the proposed device.
\end{abstract}

\section{Introduction}

The heavy-duty vehicles (HDV) represent only $4 \%$ of all the vehicles in the European Union, but they produce $30 \%$ of the carbon dioxide $\left(\mathrm{CO}_{2}\right)$ emission rates from the vehicle sources $[1,2]$. Thus, the $\mathrm{CO}_{2}$ emissions and consequently the fuel consumption of heavy trucks is much more significant issue, rather than their percentage occurrence of the overall on-road vehicles. To lower extend, but still important, the environmental impact is also affected by the tire slip in the HDVs worldwide [3]. Tire slip occurs when there is a difference between the actual distance travelled by the tire and the distance supposed to be travelled in theory. This difference occurs in the truck tires, because the lift force on the vehicle system is high, and the wheels are "floating". This effect heats up the tires, wears them out faster, and some global energy losses in the truck-trailer system might be also observed [4].

\footnotetext{
*Corresponding author: $\underline{m}$ ivanov@tu-sofia.bg
} 
Both described factors could be significantly improved with improving the aerodynamics of the heavy-duty trucks. The aerodynamic losses for a fully loaded tractortrailer are about $35 \%-55 \%$ of the overall losses for the highway environment $[5,6]$. Thus, improving the aerodynamic characteristics of the HDV-s would be the most beneficial approach, for reducing their fuel consumption and emissions.

According to the National research council in Canada, Centre for Surface Transportation Technology [7], the main areas of a tractor-trailer, which are critical for the aerodynamics of the trucks are: the tractor streamlining (approximately 15\% contribution to the aerodynamic losses), the airflow under the trailer (approx. 28\%), the airflow to the rear of the trailer (approx. 33\%) and the airflow near the tractor-trailer gap (approx. 14\%). The tractor-trailer gap is an area with large recirculation zones and high turbulent intensity, due to the main flow coming from the top of the tractor and the side winds. This gap contributes a lot to the overall drag acting on the tractor-trailer combination. Sealing the gap would eliminate its contribution, but a minimum distance between the tractor and the trailer is required due to its operational requirements. Typical tractor-trailer gaps are about 1-meter long.

Usually, the manufacturers attempt to change the flow in the gap, by mounting devices on the tractor, like side extenders. They are mounted on the back edges of the tractor to prevent the airflow coming from the top of the tractor into the gap area. Another way used is a gap splitter, which is a plate mounted in the middle of the back of the tractor, that splits the gap into two areas in order to decrease the impact of the cross winds on the drag. Also, reducing the distance between the back of the tractor and the front of the trailer is a way to reduce the drag as well. This method is limited because a certain gap should exist so that the driver would be able to turn, load and unload in different spaces.

However, little has been done into checking the active performance of the gap, when using different trailers on an aerodynamically streamlined tractor [8]. In a recent study by Tsvetanov [9, 10], it is suggested that the airfoils could be used in the automotive industry, to generate lift force in horizontal direction, when the area around them is restricted. The author describes that, in the aircraft industry the airfoil wings are used to generate lift force, required for the aircraft movement in the sky. If such a wing is rotated at 90 degrees, and still streamlined normally, its lift force is going to be horizontal and will possibly help the forward movement of a truck.

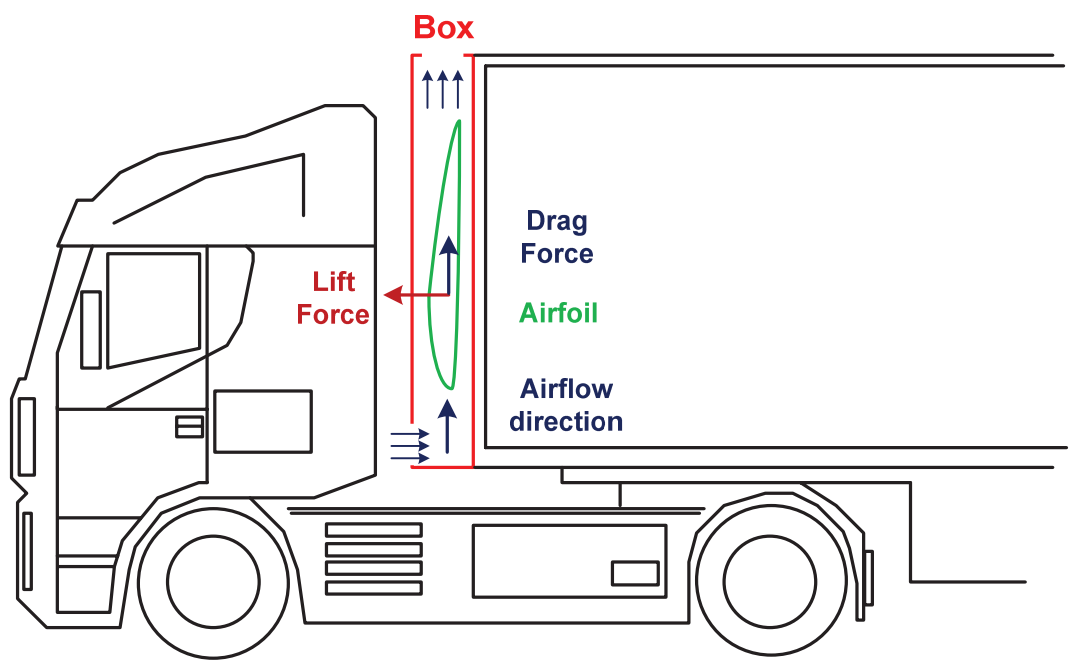

Fig. 1. Drag compensation device with air foil 
There are two main prerequisites in order to have a working system as the described above. First, the airfoil must be fixed to the vehicle. Second, there must be an air flow around it with the corresponding direction and velocity. The described idea is to have a box with an airfoil with specific characteristics inside and to mount the box vertically to the vehicle. After that, an air flow in the box must be ensured. Considering the HDV-s, the best place to mount this kind of device is the gap between the tractor and the trailer, as there is enough space and is one of the main areas of aerodynamic losses in the trucks. The described idea is presented schematically on Fig. 1. The study of Tsvetanov [10] also suggests the airfoil profile with the best performance for this application - the GOE 428 Airfoil. Considering the figure, the resulted lift force will be in the direction of the vehicle motion, i.e. opposing the drag force.

As it was mentioned, in order this system to work properly, a significant airflow around the airfoil should be ensured, and the airfoil should transfer the generated lift force to the truck-trailer system without losses. But several questions arise, like what should be the optimal size of the airfoil, in order to generate significant lift force, and how much usable flow velocity could be generated in the restricted area? The presented study deals with these questions, using numerical simulations by the methods of the CFD.

\section{Aim of the presented study}

The aim of the presented study is to analyze numerically the airfoil performance in low velocity restricted area, as part of a drag compensation device for on-road freight vehicles.

\section{Model build-up and investigated cases}

According to the main idea of the examined drag compensation device, the airfoil is positioned in a box, which restricts the air around it. For the purpose of the study, the box dimensions are selected, so that it fits the gap between the tractor and the trailer of a truck. The presented task is two dimensional, considered initially for simplification and for the further model improvements, it will be three dimensional. The size is selected after extensive literature research for the gap size of the different truck-trailer configurations. The length of the box is $2.10 \mathrm{~m}$ and the width is $0.8 \mathrm{~m}$, and it is the same for all investigated cases. The optimal airfoil size is investigated under constant velocity airflow, with the value of $28 \mathrm{~m} / \mathrm{s}$. This velocity corresponds to $100 \mathrm{~km} / \mathrm{h}$, which is the maximum allowed highway speed limit for the trucks in Bulgaria. For the purpose of the study, the box is significantly simplified, and no flow directing elements are considered, for the inlet and outlet sections of the device. This means that, much lower performance of the device is expected in reality.

Table 1 shows the all 10 investigated cases, with the airfoil scales and the leading-edge position coordinates. In the $100 \%$ Case (see Fig. 2), the airfoil is the largest that can fit in the selected box, and the length of the wing is $2 \mathrm{~m}$. Respectively, in the $10 \%$ case and the profile is $0.2 \mathrm{~m}$ long. As it can be seen from Fig. 2 with the decrease of the size of the airfoil, the amount of air flowing around it increases. Comparing the $100 \%$ Case and the $10 \%$ Case it can be concluded that the air around the last case is significantly more, but the profile is a lot smaller and the lift force that it generates is smaller. In all cases the airfoil is positioned in the center of the box.

The four sides of the restricting box are named as follows: inlet, outlet, upper wall and lower wall. The flow direction is from the inlet cross-section to the outlet cross-section. As it was mentioned, no directing devices at the inlet and at the outlet cross-sections are considered in this study. 
Table 1. Airfoil position and size scale in the investigated cases

\begin{tabular}{|c|c|c|c|c|c|c|}
\hline \multirow{2}{*}{ Case } & \multirow{2}{*}{ Scale } & \multicolumn{4}{|c|}{ Leading edge position (m) } & \multirow{2}{*}{$\begin{array}{c}\text { Airfoil length } \\
(\mathrm{m})\end{array}$} \\
\hline & & inlet & outlet & upper wall & lower wall & \\
\hline 1 & $100 \%$ & 0,05 & 2,05 & 0,5 & 0,3 & 2 \\
\hline 2 & $90 \%$ & 0,15 & 1,95 & 0,5 & 0,3 & 1,8 \\
\hline 3 & $80 \%$ & 0,25 & 1,85 & 0,5 & 0,3 & 1,6 \\
\hline 4 & $70 \%$ & 0,3 & 1,8 & 0,49 & 0,31 & 1,4 \\
\hline 5 & $60 \%$ & 0,4 & 1,7 & 0,48 & 0,32 & 1,2 \\
\hline 6 & $50 \%$ & 0,5 & 1,6 & 0,47 & 0,33 & 1 \\
\hline 7 & $40 \%$ & 0,6 & 1,5 & 0,43 & 0,37 & 0,8 \\
\hline 8 & $30 \%$ & 0,75 & 1,35 & 0,42 & 0,38 & 0,6 \\
\hline 9 & $20 \%$ & 0,8 & 1,3 & 0,41 & 0,39 & 0,4 \\
\hline 10 & $10 \%$ & 0,9 & 1,2 & 0,4 & 0,4 & 0,2 \\
\hline
\end{tabular}

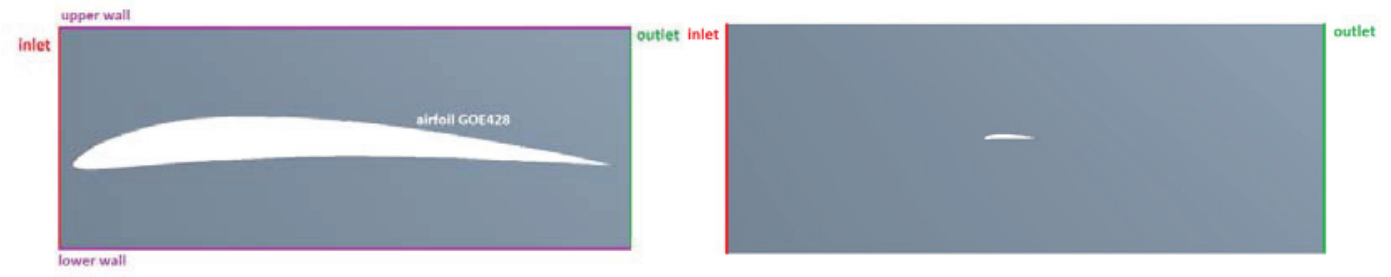

Fig. 2. Model geometry for Case $1-100 \%$ and for Case $10-10 \%$

\section{Numerical model and initial and boundary conditions}

ANSYS Fluent software package is used for the steady-state numerical simulations in the presented study. The turbulent model is the standard $\mathrm{k}-\varepsilon$, with the SIMPLE algorithm for correct linkage between pressure and velocity. The fluid in the domain is defined as air with density $1.225 \mathrm{~kg} / \mathrm{m} 3$, and initial constant velocity of $28 \mathrm{~m} / \mathrm{s}$, from the inlet section. The solid areas are the airfoil and the walls. The material used for them is aluminum, with density: $2719 \mathrm{~kg} / \mathrm{m} 3$ and roughness constant 0.5. Standard wall functions are used for the solid surfaces.

The computational grid consists of triangular elements for all investigated cases, which gives possibility for refinement in the boundary layer and better options for resizing of the elements for the different airfoil sizes. In Table 2 are shown the grid characteristics, for all the cases observed. The minimum size of the cells is used around the airfoil. The sizing of the elements on the edge of the profile is the corresponding minimal size from the table. The sizing on the walls of the box is $0,001 \mathrm{~m}$ for all cases. The maximum size is used at the areas with less velocity gradients. The total number of the elements outcomes from the grid sizing implemented in the model. Form preliminary tested grid independence solution, it was found out that grids bigger than 80000 elements satisfy the accuracy requirements of the problem, since the case is relatively simple.

Figure 3 shows the computational grid of the Case 1, and Case 10, respectively. As seen, the grid is finer on the edges of the airfoil and the walls of the box and coarser in the middle areas. 
Table 2. Computational grid characteristics for the investigated cases

\begin{tabular}{|c|c|c|c|c|}
\hline Case & Scale & Number of elements & Max cell size (m) & Min cell size (m) \\
\hline $\mathbf{1}$ & $100 \%$ & 106562 & 0,05 & 0,001 \\
\hline $\mathbf{2}$ & $90 \%$ & 101947 & 0,05 & 0,001 \\
\hline $\mathbf{3}$ & $80 \%$ & 97016 & 0,05 & 0,001 \\
\hline $\mathbf{4}$ & $70 \%$ & 90766 & 0,05 & 0,001 \\
\hline $\mathbf{5}$ & $60 \%$ & 84752 & 0,05 & 0,001 \\
\hline $\mathbf{6}$ & $50 \%$ & 105793 & 0,05 & 0,0005 \\
\hline $\mathbf{7}$ & $40 \%$ & 97894 & 0,03 & 0,0005 \\
\hline $\mathbf{8}$ & $30 \%$ & 87846 & 0,03 & 0,0005 \\
\hline $\mathbf{1 0}$ & $20 \%$ & 100528 & 0,01 & 0,0005 \\
\hline
\end{tabular}
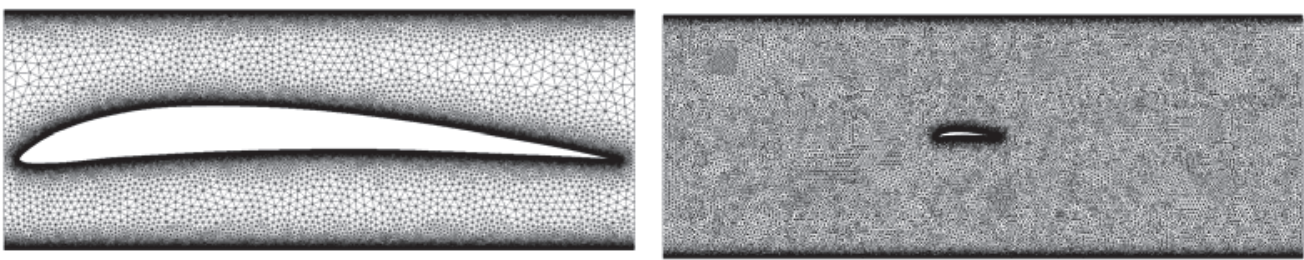

Fig. 3. Computational grid for Case $1-100 \%$ and for Case $10-10 \%$

\section{Numerical Results}

On Fig. 4 is shown the velocity distribution profile, for Case 1. The maximum velocity of the flow is $54 \mathrm{~m} / \mathrm{s}$ and the minimum is $0 \mathrm{~m} / \mathrm{s}$ at the boundary layer and the stagnation point at the leading edge of the profile. It increases to $54 \mathrm{~m} / \mathrm{s}$ at the top of the airfoil, which is almost double the initial velocity. At the bottom of the profile the velocity is around $40 \mathrm{~m} / \mathrm{s}$, which is also higher than the initial velocity. At the tale of the airfoil, decrease of the velocity is observed as it reaches $10 \mathrm{~m} / \mathrm{s}$. The boundary layer is thinner at the beginning of the box and wider at the end of the box. The lift force in this case is $849.51 \mathrm{~N}$. The drag force is $42.43 \mathrm{~N}$.

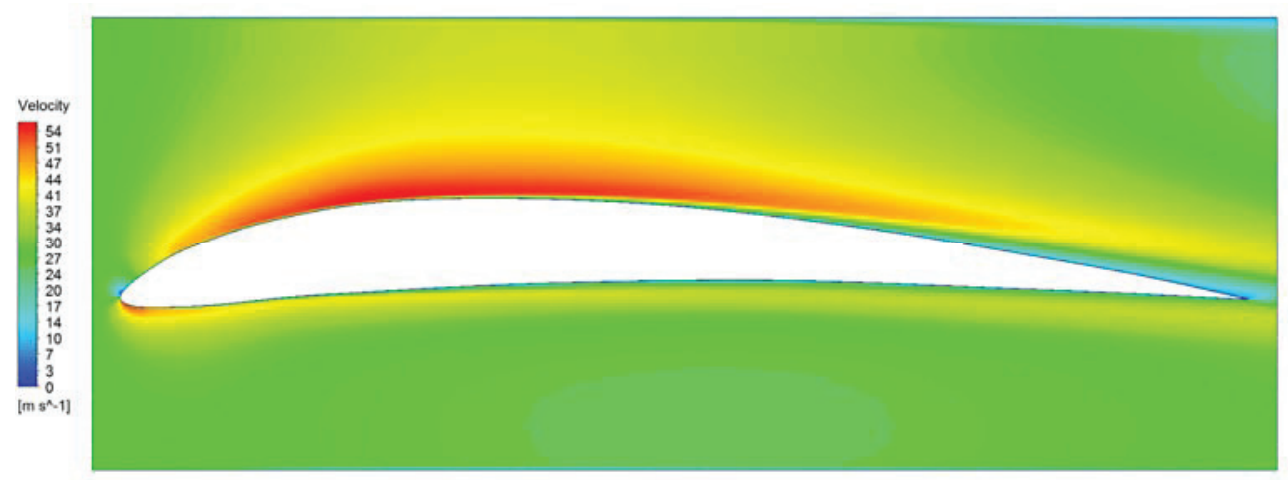

Fig. 4. Velocity distribution for Case $1-100 \%$ 


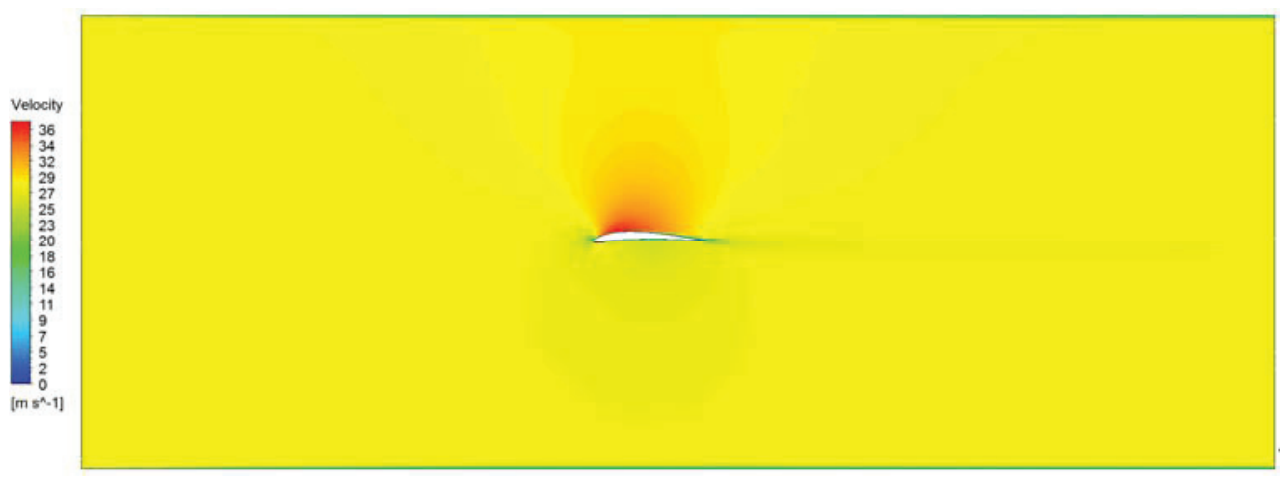

Fig. 5. Velocity distribution for Case $10-10 \%$

Respectively, on Fig. 5 is shown the velocity distribution profile, for Case 10. The maximum velocity of the flow is $36 \mathrm{~m} / \mathrm{s}$ and the minimum is again $0 \mathrm{~m} / \mathrm{s}$ at the boundary layer and the stagnation point at the leading edge of the profile. At the bottom of the profile the velocity is around $25 \mathrm{~m} / \mathrm{s}$. At the tale of the airfoil, decrease of the velocity is observed as it reaches $16 \mathrm{~m} / \mathrm{s}$. The boundary layer is again thinner in the beginning of the box and wider at the end of the box. The lift force in this case is $47.78 \mathrm{~N}$. The drag force is $1.51 \mathrm{~N}$.

Table 3 summarizes the numerical results for all studied cases, and the graphical results for the lift force and the drag force are shown on Fig. 6 and Fig. 7. As it can be seen, the values for the lift force and the drag force decrease linearly and exponentially with the decreasing of the size of the airfoil. Case 1 is the one in which the highest forces are calculated, which is expected since the airfoil is with the biggest size. The results for the $100 \%$ Case are similar to the values described in the paper of Tsvetanov [10], who suggests generated lift force for this size and velocity, equal to $850 \mathrm{~N}$, calculated by numerical software.

Table 3. Lift and drag force results for the investigated cases

\begin{tabular}{|c|c|c|c|c|}
\hline Case & Scale & Lift Force (N) & Drag Force (N) & Airfoil length (m) \\
\hline $\mathbf{1}$ & $100 \%$ & 849,51 & 42,43 & 2 \\
\hline $\mathbf{2}$ & $90 \%$ & 724,06 & 19,07 & 1,8 \\
\hline $\mathbf{3}$ & $80 \%$ & 604,91 & 12,93 & 1,6 \\
\hline $\mathbf{4}$ & $70 \%$ & 530,92 & 10,24 & 1,4 \\
\hline $\mathbf{5}$ & $60 \%$ & 421,67 & 7,87 & 1,2 \\
\hline $\mathbf{6}$ & $50 \%$ & 321,69 & 5,57 & 1 \\
\hline $\mathbf{7}$ & $40 \%$ & 242,5 & 4,37 & 0,8 \\
\hline $\mathbf{8}$ & $30 \%$ & 168,87 & 3,01 & 0,6 \\
\hline $\mathbf{9}$ & $20 \%$ & 102,69 & 2,16 & 0,4 \\
\hline $\mathbf{1 0}$ & $10 \%$ & 47,78 & 1,51 & \\
\hline
\end{tabular}




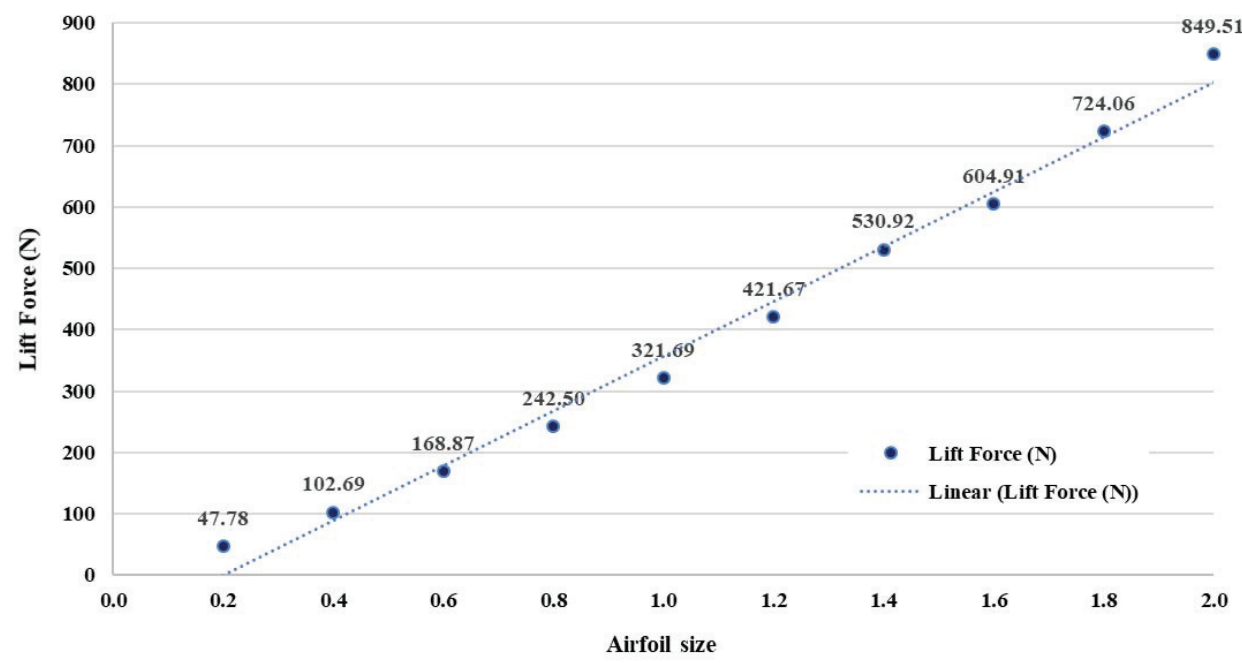

Fig. 6. Lift force results, in relation with the airfoil length in the restricted area

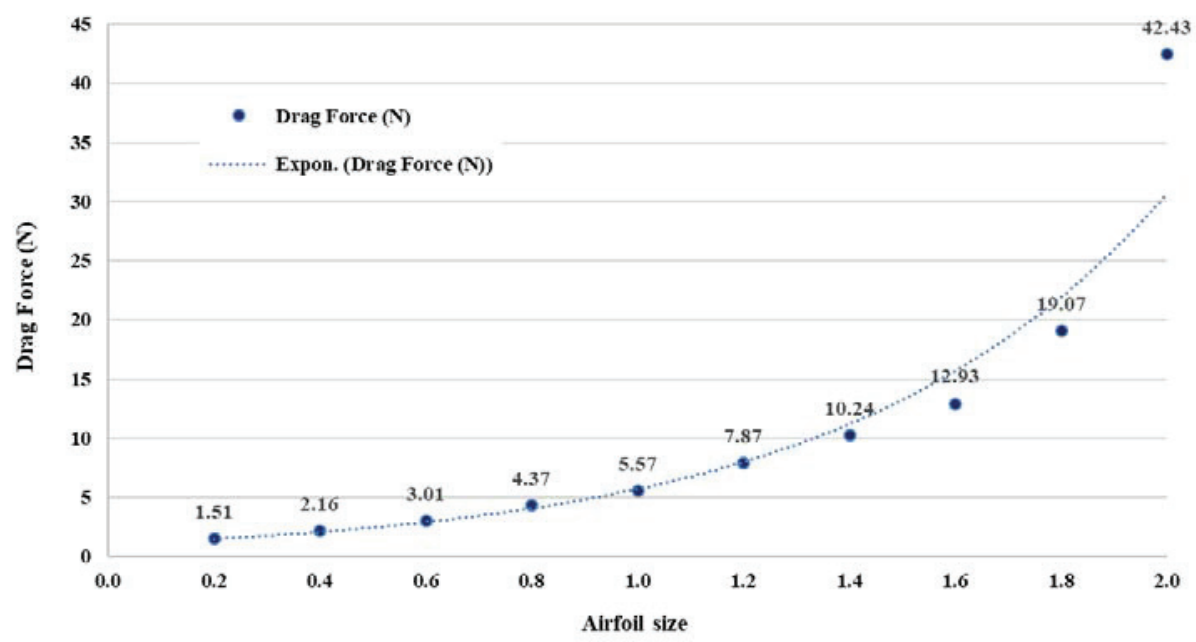

Fig. 7. Drag force results, in relation with the airfoil length in the restricted area

\section{Model limitations}

The described model characterizes the ideal case, in which the boundary and initial conditions are flawless to achieve the maximum result desired. But the model also has several important limitations that must be considered in further studies, in order to achieve the most accurate result.

The presented simulations are performed in two-dimensional mode, and consequently the turbulence effect cannot be fully analyzed. Three-dimensional simulations will give more detailed and accurate results and will provide better understanding of the problem. Correspondingly, the impact of the width of the airfoil over the resulting lift and drag forces will be assessed. Also, the modelled surface effects (shear stresses and pressure distribution) are largely affecting the boundary layer over the airfoil, which is not well 
accomplished by the selected standard k-e model. Further simulations with the more advanced $\mathrm{k}-\omega$ model could be preferable, as well as further refinement of the computational grid in the boundary layer.

In this work, the mounting of the airfoil in the suggested box is not specified. The fixture to the box walls will significantly influence the results obtained. Another model limitation, which should be considered, is the different possibilities for the materials used, for the airfoil and the box. Their characteristics like surface roughness, density and elasticity will have an impact over the velocity and pressure profiles over the streamlined surfaces, and also will influence the generated and transferred lift and drag force. Temperature is a significant factor too and might change the flow parameters in the box. In the presented simulations the temperature factor is not considered.

Finally, the design of the inlet and outlet sections of the box are not discussed in the paper, and the presented results show, that they will have the most important impact over the performance of this drag compensation device. There are huge limitations for the design of the inlet section, coming from the truck manufacturer's design constraints. The ideal case with airflow velocity of $28 \mathrm{~m} / \mathrm{s}$ is very challenging for the design realization of the presented idea and should be studied further.

\section{Conclusions}

The presented study investigates the aerodynamic performance of airfoil GOE428 in low velocity restricted area, designed as an aerodynamic loss compensation device, for freight transport vehicles. It focuses on the lift and drag force generation for different airfoil sizes, at constant flow velocity. The main methods used, are based on the Computational Fluid Dynamics tools.

For the described model conditions, the idea potential of a working airfoil in a restricted area is proved. The $2 \mathrm{D}$ simulation results show that the lift force is normally generated in the restricted area, and the values increase with the increase of the size of the profile.

The biggest airfoil size has generated the biggest value for the lift force correspondingly. The resulted lift force for this case is $849.51 \mathrm{~N}$, which is confirmed value from previously published research studies.

The highest obtained value of the lift force seems that might not have significant effect on the aerodynamic characteristics of the truck, compared with the weight of a typical tractor-trailer vehicle. Also, in this study the mechanical characteristics of the box are not studied, neither is the way of transferring the lift force into mechanical energy that could help the aerodynamics of the heavy-duty vehicles.

It is considered that all the above conclusions and the presented important model limitations, will assist the further development of the proposed aerodynamic loss compensation device.

The presented study is supported by "RDS" at TU-Sofia, as part of the activities under the "Perspective leaders" project, with Contract 201 0018-02, entitled: "Experimental assessment of low velocity air jets, for boundary and initial conditions in numerical modelling studies".

\section{References}

1. R. Muncrief and Sharpe B. Overview of the heavy-duty vehicle market and $\mathrm{CO} 2$ emissions in the European Union, Int. Council on Clean Transportation ed. 2015-6, (2015) 
2. European Automobile Manufacturers' Association (ACEA) Report: "Vehicles in use Europe”, https://www.acea.be/publications/article/report-vehicles-in-use-europe-2017, (2017)

3. F. Browand, "Reducing aerodynamic drag and fuel consumption", Aerospace and Mechanical Engineering lect. course Oct. 2005, Viterbi School of Engineering, Univ. of South. California, (2005)

4. H. Chowdhury, Loganathana B., Mustary I., Moriab H. and Alama F. "Effect of various deflectors on drag reduction for trucks”, J. Energy Proc. Vol. 110, pp. 561566, doi: 10.1016/j.egypro.2017.03.185, (2017)

5. D. Huang, J. Li and Y. Liu, "Airfoil dynamic stall and aeroelastic analysis based on multi-frequency excitation using CFD method”, J. Proc. Eng., Vol. 99, pp. 686-695, doi: 10.1016/j.proeng.2014.12.590, (2015)

6. M. Metka, “An examination of active drag reduction methods for ground vehicles", Undergraduate Thesis, Dep. of Mech. Eng., The Ohio State University, (2013)

7. J. Patten, B. McAuliffe, W. Mayda and Tanguay "Review of aerodynamic drag reduction devices for heavy trucks and buse”, Nat. research council of Canada, Centre for surface transp. tech., rep. 54-A3578, (2012)

8. A. Shukri and M. Akram, "Improvement of aerodynamics characteristic of heavy trucks”, proc. 3rd Int. Conf. on Trends in Mech. and Ind. Eng. Kuala Lumpur, pp. 246255, (2013)

9. Y. Tsvetanov, "Vehicles and aerodynamics - trends and problems", Sci. works of Univ. of Ruse, SAT-9.3-1-HP-12, vol. 55, pp 181-187, (2016)

10. Y. Tsvetanov, A. Terziev, "Characterization of a wing profile for aerodynamic compensation of road vehicles”, Proc. Int. Conf. FPEPM at TU-Sofia, ISSN 1314 5371, Vol. II, pp.29-36, (2017) 